\title{
A robot-based burr measurement system for the automotive ind ustry
}

\author{
F. Benati, C. Butler, S. Gatti, F. Sacerdotti, Q. Yang \\ Department of Manufacturing and Engineering Systems, Brunel University, Uxbridge \\ (Middlesex), UK
}

\begin{abstract}
Burrs are often difficult to detect and measure because of their intrinsic variability in shape and dimension. No automotive standard had been established about their acceptable dimensions and measurement techniques for sheet steel products. For the automotive industry, even burrs of the size of $100 \mu \mathrm{m}$ are perceived as damaging because of their dramatic impact upon panel corrosion resistance and assembly performance. It is critical to measure burrs during panel manufacture in order to control the process. The characterization of the typical burr produced has been carried out employing $3 D$ measurements with a surface profilometer and a SEM. This analysis has shown a typical triangular burr shape and some characteristic dimensions. A contact method and two laser-triangulation systems have been developed. The instrument accuracy was analyzed, based upon a full factorial experimentation over a set of typical panels edges.
\end{abstract}

\section{Introduction}

Blanking is probably the most used operation in the sheet metal working. Every product has to be blanked, either to cut exceeding material after a deep drawing process or just to punch a hole in the panel. This operation aims to reproduce the perfect shearing process, but since the working conditions are far from the ideal process (material shear without bending) it develops a true triaxial stress system [1].

The operation consists of a punch that penetrates the sheet up to a third of the thickness then the material usually collapses causing the slug to break off. When the slug breaks off, the panel tears it away and, in doing that, it produces the burr [2].

There are many techniques already available on the market suitable for detecting burr presence over a manufactured object. The automotive industry is striving to prevent burr formation because of the impact upon assembly performance and quality customers perceive. It has become necessary to move over the simple detection to implementation of suitable sensors for measuring burrs critical dimension [3]. Laboratory measurements are quite straightforward since many techniques can be applied (e.g. Surface Profilometer, SEM). These techniques provide highly accurate visualisation of burr size, but for a number of reasons, they cannot be applied on the shop floor.

Typically, the reasons are the following:

- Burr Type: autobody panel burrs occur along edges and around any hole and so they do not measure the same point every time.

- Environment: the surrounding of the production line is not ideal for taking high accuracy measurements. Vibrations are introduced by many sources (from Robot movements to the presses producing panels).

- Speed: many laboratory-based systems produce very high accuracy but require an evaluation time far too long for the shop floor inspections.

\section{Burr Characterisation}

- Longitudinal Section. The burrs can assume many shapes, depending on its formation process, for instance [4]:

Blanking - Triangular shape

Fine Blanking - Thin rectangular shape

Milling - Rectangular or triangular

Laser Cutting - Globular

The characteristic longitudinal dimension for burrs that was found during this framework is the following:

Surface Roughness (Ra) $\quad 1.4 \mu \mathrm{m}$

Burr Height

$50-350 \mu \mathrm{m}$

Burr Width 


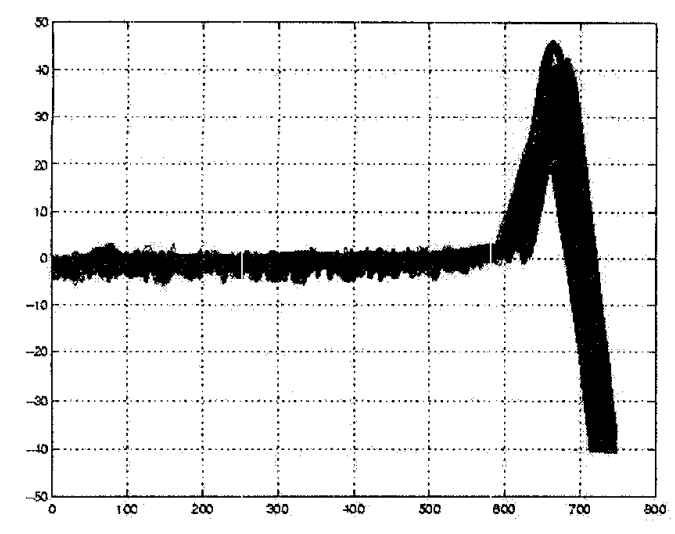

Figure 1 - Longitudinal Section of a burr. This plot was obtained by over-drawing 256 burrs profiles from a 3D profilometer measurement.

- Transversal Section. The main feature noticed was that burrs from blanking were highly irregular in profile [5].

It was found that burr edges show the same characteristics of raw material roughness.
Surface Spacing (Sm)
Burr edge Spacing
Surface Roughness (Rz)
$50-200 \mu \mathrm{m}$
Burr edge Peak-Valley

$$
\begin{aligned}
& 50-200 \mu \mathrm{m} \\
& 5-10 \mu \mathrm{m}
\end{aligned}
$$$$
5-20 \mu \mathrm{m}
$$

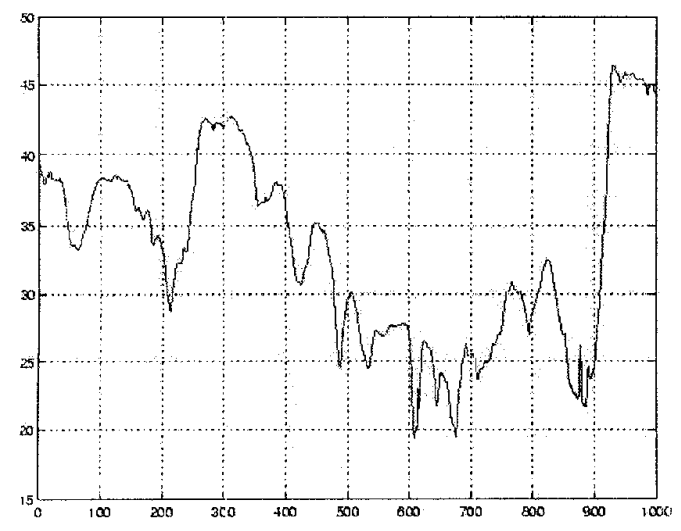

Figure 2 - Transversal Section of a burr. The variability of burr's edge is of the same order of the surface roughness. A secondary undulation

(longer than $1 \mathrm{~mm}$ period) derives from the blanking process.

Making a single measurement of a burr generally will not allow repeatable results or establish trends. Often the best solution involves combining two or more inspection techniques [6].

\section{Available Instruments to measure Burrs}

The most natural way of detecting burr is to use a finger therefore contacts methods most closely resemble and may appear an ideal choice. Due to the precision required by the measurement, they are usually very small and delicate [7]. On the other hand, the advantages of non-contact techniques are considerable: they do not show wear on both the instrument and the burr (which is common with contact instruments) and can access difficult geometry (such as recessed holes).

\subsection{Laboratory Techniques}

- Mechanical profilometers allows measurement of small excursions from nominal value. It is reasonably accurate and the burr does not get bent during the tracing operation. This technique is heavily time consuming and, especially if it needs a 3D characterisation of the burr, many problems can be experienced during the measurement because of the probe crashing against the burr on the return.

Therefore, this technique gives an excellent reference to evaluate the performance of other techniques.

- SEM gives both image and dimension of the burr. It is very useful during the stage of burr characterisation because of the precision and the visual appearance. Using this technique it has been possible to detect very small defects, burr bending and so on. This method is also very time consuming and is suitable only for small parts because of its use of vacuum.

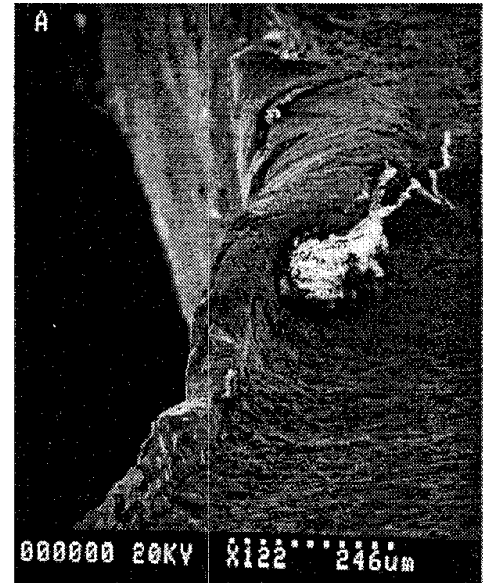

Figure 3-SEM images of a burr. It is possible to recognise some human skin probably scratched by the burr during panel handling. 


\subsection{Suitable On-line Techniques}

It was decided to develop and test some suitable techniques for on-line burr inspection. The chosen techniques are the following.

- Beam Deflection is the natural simplification of the mentioned profilometers. The general principle is to measure the deflection of a probe contacting the panel and, by sliding over the edge, it possible to obtain burr height.

The developed probe is based on a set of two mild steel cantilevers (Young modulus $210 \mathrm{Mpa}$ for a maximum load of $0.3 \mathrm{~N}$ ) clamped on a basement. On the edge of the cantilevers it was fit a bearing ball ( $3 \mathrm{~mm}$ diameter) to minimise wear and assure correct contact with the panel before the edge. Unfortunately, in the situation of non-perfect alignment between panel and probe, such a diameter may lead to consistent error (that can be calculated with easy trigonometry). For this reason it was decided to employ two probes instead of in order to measure the inclination between panel and probe and, with that, correct the measured burr height.

The beams are fitted with strain gauges on both sides for better temperature compensation and half bridge amplification. This system enables a measurable range of $2 \mathrm{~mm}$ with $0.5 \mathrm{Volt} / \mathrm{mm}$ output signal.

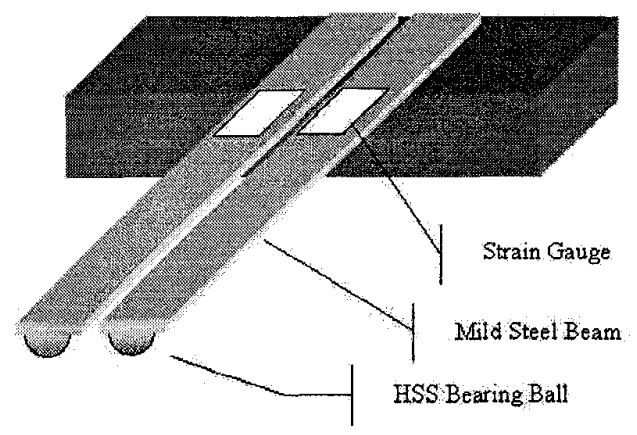

Figure 4 - Contact Probe based upon beam deflection.

- Triangulation Displacement Meters use a light beam projected on to the surface to be measured. The diffuse reflection of the beam is then captured by a position-sensing device through a lens system.

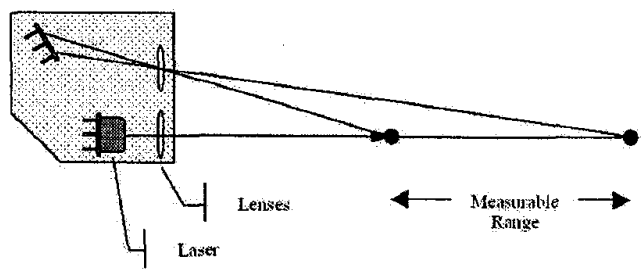

Figure 5 - Laser Triangulation Sensor operation.

As the sample moves relative to the beam within the measurable range, the reflected light fluctuates and the position on the array varies. The positional array provides a voltage output that can be calibrated to be proportional to the surface displacement. Two different laser triangulation sensors were used: the first uses a digital CCD and the second uses an analog Position Sensitive Detector (PSD) to convert from laser deflection to displacement.

An important concern was the spot size (ideally, it should be smaller than the feature to detect). The spot size is, for both the systems, of the order of $100 \mu \mathrm{m}$ (which is also the average burr's width).

The CCD system has a displacement range of \pm 5 $\mathrm{mm}$ over an output voltage range of \pm 5 Volt with a resolution of $1 \mu \mathrm{m}$.

The PSD system has a bigger displacement range $( \pm 10 \mathrm{~mm}$ ) over the same voltage range and the same (declared) resolution.

\section{Experiments}

To test the instruments before the installation in the work environment the most important influencing factors were reproduced in laboratory with full factorial experimentation. Instrument accuracy was tested against surface condition (oil presence), motion speed and two typical burrs from the burr characterisation framework. The experimental design was the following:

\begin{tabular}{l|cc} 
Factor & \multicolumn{2}{|c}{ Levels } \\
\hline Oil Presence & Yes & No \\
Burr Type & A & B \\
Velocity & $0.05 \mathrm{~mm} / \mathrm{s}$ & $5 \mathrm{~mm} / \mathrm{s}$
\end{tabular}

\subsection{Panel Surface Conditions}

During pressing, the panels are usually exposed to a variety of lubricants used both to aid forming and to protect the dies. Different grades of steel and aluminum may be used to produce different type of 
parts such as structural and aesthetic panels. Two oil types were identified as being likely to be present on the panel immediately after pressing (Quaker E5009 and PM86). The amount varies but it was generally a thin film of the order of $1.5 \mathrm{~g} / \mathrm{m}^{2}$. Both oil types were used and artificially applied to panels during the trials to assess the effect. The surface texture of the panels produced was not considered to have caused any notable changes in measurements. Although in preliminary trials, panel reflectivity was found of critical importance. Heavily dusted surfaces and corroded areas were found affecting laser focus because of their different color from the rest of the panel. In order to minimise this effect the tested panels had to be chosen in good corrosion conditions and properly cleaned.

\subsection{Experiments Preparation}

The burr samples were first washed with a suitable degreaser and then clamped to the Coordinate Measurement Machine (CMM) basement. Lubricants were added manually when required and cleaned after the measurements. Speed and starting position were set using the standard software of the CMM. The displacement signal was sampled using a data acquisition board equipped with ad-hoc software developed internally, in order to assure a linear resolution of 10010 points $/ \mathrm{mm}$. The signal was first filtered (only for optical systems since the contact probe is naturally ball-filtered) from noise by employing a moving average over ten points. The profile was then linearised and burr height was simply calculated as the maximum value. The contact probe output was further processed by correcting for the inclination error.

\section{Results Analysis}

Oil presence has shown important only for the more accurate optical system (CCD), in terms of decreasing the real burr high of the same order of the oil lying on the flat area. PSD laser system did not show any effect of oil probably because covered by its lower accuracy.
Main Effects Plot - Data Means for Burr

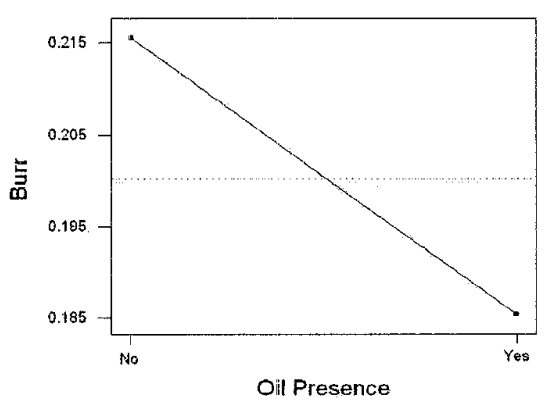

Figure 6 - Lubricant effect on CCD laser system (all measurements in $\mathrm{mm}$ ).

The contact probe was only sensitive to the sliding speed, in terms of both final value and reading variability.

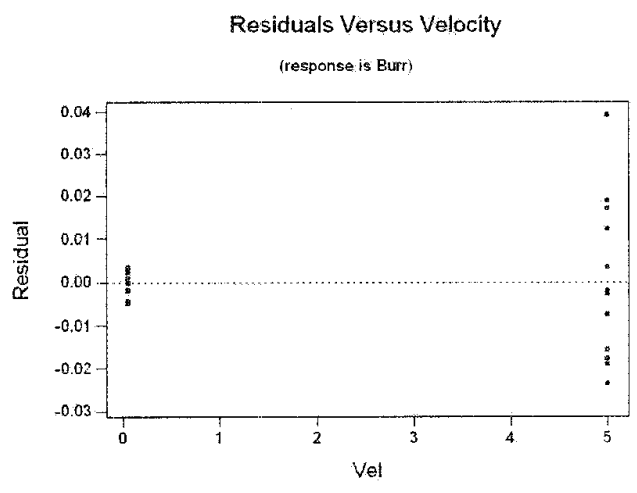

Figure 7 - Effect of sliding velocity for the contact probe (all measurements in $\mathrm{mm}$ ).

A Tukey $95 \%$ confidence interval for burr height was calculated and, on the base of this, it was obtained the instrument accuracy:

Optical and contact instruments accuracy appear to be almost equivalent in terms of accuracy after data filtering.

$\begin{array}{ll}\text { Instrument } & \text { Accuracy } \\ \text { LDS Keyence } & \pm 10 \mu \mathrm{m} \\ \text { LDS Matsushita } & \pm 25 \mu \mathrm{m} \\ \text { Contact Probe } & \pm 13 \mu \mathrm{m}\end{array}$

\section{Conclusions and Discussion}

It is critical to measure burrs during panel manufacture in order to control the process. The 
characterization of burrs from the autobody panels manufacturing process has shown a typical triangular burr shape and some characteristic dimensions. A contact method and two laser-triangulation systems have been developed.

The instrument accuracy was analyzed, based upon a full factorial experimentation over a set of typical panels edges. It was found that all three instruments can, virtually, identify correctly burr's height with the above mentioned accuracy.

Optical instruments have the advantage of not showing any wear but have shown a very high noise due to sensitivity to surface topography, lubricant applied on the surface and reflectivity of the surfaces.

The contact probe has shown very low sensitivity to the mentioned surface characteristic because of the natural filtering done by the bearing ball but the measurable range was sensibly less than the optical one ( $2 \mathrm{~mm}$ versus $\pm 5 \mathrm{~mm}$ ).

The main concerns originating from this experimentation work are related to the algorithm to use to evaluate burr height. The recognition of the flat area before the burr over which linearise the entire signal is easy with human supervision. To devise a robust algorithmic recognition of the mentioned area applicable in working environment could be very difficult and such an instrument would still probably require human interaction.

\section{References}

[1] K. Lange

Handbook of Metal Forming

Mac Graw Hill 1985

[2] A.R. Tressel

In Plant Standard on Burrs

SME Technical Paper MR77 - 467, 1977

[3] American Metal Stamping Association,

Technical Research and Standards Committee

Metal Stamping Design Guide -

Data Sheet No. 2022, 31/01/1970

[4] LaRoux K. Gillespie

The Battle of the Burr

Manufacturing Engineering, 1996

[5] S. Mia

Autobody Panel Burr Measurement System

4M Main Project Report, 1998

[6] LaRoux K. Gillespie Inspecting for Burrs

Manufacturing Engineering, April 1998

Vol. 120 , N. 4, pp. $70-76$

[7] LaRoux K. Gillespie

The Battle of the Sheetmetal Burr: New

Strategies and New Tricks, 1996

http://www2.kctera.net/deburring/
General guideline of German Institute of Engineers on: Quality of cut Faces of (sheet) metal parts after cutting, blanking, trimming or piercing, May 1994 